\title{
Morphological and physiological evidence of a synaptic connection between the lateral parabrachial nucleus and neurons in the $A 7$ catecholamine cell group in rats
}

\author{
Chia-Yi Liu ${ }^{1,2+}$, Meng-Lam Lee ${ }^{3,4+}$, Chi-Sheng Yang ${ }^{5}$, Chuan-Mu Chen ${ }^{1,6}$, Ming-Yuan Min ${ }^{7}$ and Hsiu-Wen Yang ${ }^{3,4^{*}}$ (D)
}

\begin{abstract}
Background: The descending noradrenergic (NAergic) system is one of the important endogenous analgesia systems. It has been suggested that noxious stimuli could activate descending NAergic system; nevertheless, the underlying neuronal circuit remains unclear. As NAergic neurons in the A7 catecholamine cell group (A7) are a part of the descending NAergic system and the lateral parabrachial nucleus (LPB) is an important brainstem structure that relays ascending nociceptive signal, we aimed to test whether LPB neurons have direct synaptic contact with NAergic A7 neurons.
\end{abstract}

Results: Stereotaxic injections of an anterograde tracer, biotinylated dextran-amine (BDA), were administered to LPB in rats. The BDA-labeled axonal terminals that have physical contacts with tyrosine hydroxylase-positive (presumed noadrenergic) neurons were identified in A7. Consistent with these morphological observations, the excitatory synaptic currents (EPSCS) were readily evoked in NAergic A7 neurons by extracellular stimulation of LPB. The EPSCs evoked by LPB stimulation were blocked by CNQX, a non-NMDA receptor blocker, and AP5, a selective NMDA receptor blocker, showing that LPB-A7 synaptic transmission is glutamatergic. Moreover, the amplitude of LPB-A7 EPSCs was significantly attenuated by DAMGO, a selective $\mu$-opioid receptor agonist, which was associated with an increase in paired-pulse ratio.

Conclusions: Taken together, the above results showed direct synaptic connections between LPB and A7 catecholamine cell group, the function of which is subject to presynaptic modulation by $\mu$-opioid receptors.

Keywords: Lateral parabrachial nucleus, A7, Nociception, Catecholamine

\section{Background}

Norepinephrine (NE) is an important neuronal modulator in the brain and plays significant roles in the regulation of many brain functions, including pain modulation [1]. Intrathecal injection of $\mathrm{NE}$ or $\alpha-2$ receptor agonists results in dramatic analgesia in rats [2], the mechanisms underlying which have been shown to involve inhibitory effect of NE on nociceptive neurons

\footnotetext{
*Correspondence: hwy@csmu.edu.tw

${ }^{\dagger}$ Equal contributors

${ }^{3}$ Department of Biomedical Sciences, Chung Shan Medical University, 110 Chien-Kuo N. Rd, Sec. 1, Taichung 402, Taiwan

${ }^{4}$ Department of Medical Research, Chung Shan Medical University, Taichung, Taiwan

Full list of author information is available at the end of the article
}

located in the substantia gelatinosa area of the dorsal horn through activation of $\alpha 2$-adrenoceptors [1-3]. As there are no noradrenergic (NAergic) neurons in the dorsal horn of the spinal cord [4], it is generally believed that principal supply of NAergic innervation to the dorsal horn arises from the locus coeruleus (LC) (also referred to as the A6 catecholamine cell group) and the A7 catecholamine cell group (A7) located, respectively, in the dorsomedial and dorsolateral pons [5-8]. Direct stimulation of the A7 area results in an antinociceptive effect and the effect is blocked by intrathecal injection of $\alpha-2$ receptor antagonists, showing that NAergic A7 neurons are indeed involved in

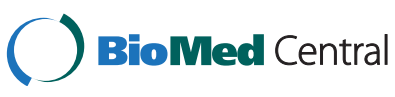

(c) 2015 Liu et al. Open Access This article is distributed under the terms of the Creative Commons Attribution 4.0 International License (http://creativecommons.org/licenses/by/4.0/), which permits unrestricted use, distribution, and reproduction in any medium, provided you give appropriate credit to the original author(s) and the source, provide a link to the Creative Commons license, and indicate if changes were made. The Creative Commons Public Domain Dedication waiver (http://creativecommons.org/publicdomain/zero/1.0/) applies to the data made available in this article, unless otherwise stated. 
intrinsic analgesia mediated by NE acting on $\alpha-2$ receptors at the spinal cord level $[9,10]$.

The intrinsic NAergic pain regulatory system in the spinal cord has low tonic activity [11]. In support of this argument, recent studies of slice preparations have shown that NAergic A7 neurons spontaneously fire action potentials at a very low frequency $(\sim 0.5 \mathrm{~Hz})$ when the fast synaptic transmissions are blocked [12, 13]. Furthermore, the spontaneous firing rate of NAergic A7 neurons is very sensitive to bath application of substance-P $[12,14]$ and $\mathrm{GABA}_{B}$ receptor antagonists [15], showing that synaptic drives are needed for operation of this system. In vivo studies have shown that peripheral noxious stimuli causes an early phase excitation of NAergic LC neurons, followed by a late phase of prolonged inhibition [16]; it also enhances NE release in the dorsal spinal cord $[17,18]$. These observations suggest that ascending nociceptive signal might be a possible source of synaptic drive for activating NAergic neurons in the pons. Together with the fact that NE modulates nociceptive neurons in the spinal cord, it is likely that pontine NAergic neurons and dorsal horn neurons have reciprocal connections and that such reciprocal connections could function as a negative feedback system to inhibit the ascending nociceptive signal by causing NE release at the spinal level. However, the neuronal circuits that are responsible for the operation of this negative feedback control system remain unclear. For example, it is yet uncertain whether there are direct reciprocal projections between pontine NAergic and dorsal horn neurons, or relay by other nuclei in the brainstem is involved. A potential candidate that can relay the nociceptive signal to pontine NAergic neurons is the lateral parabrachial nucleus (LPB), which receives direct synaptic connections from dorsal horn neurons and transfers the nociceptive signal from peripheral to many painrelated areas in the brain [19-21]. Accordingly, the aim of this study is to test whether pontine NAergic neurons receive direct synaptic input from $L P B$.

\section{Methods}

\section{Tracer injection}

The use of animals in this study was in accordance with the rules for animal research of the Ethical Committee of Chung-Shan Medical University. Male Sprague-Dawley rats weighing 200-300 g were anaesthetized with $5 \%$ isoflurane in pure oxygen. A small craniotomy was made, the dura was reflected, and a total of $0.3 \mu \mathrm{l}$ of $10 \%$ biotin dextran amine (BDA: MW 10,000; Invitrogen, Carlsbad, $\mathrm{CA}$ ) in saline were injected via a 29-gauge stainless steel needle tilted 28 degrees to the vertical into the LPB of the left brainstem at the following coordinates: $5.30 \mathrm{~mm}$ posterior to the bregma, $1.9 \mathrm{~mm}$ lateral to the midline, 6.9 $\mathrm{mm}$ ventral to the cortical surface [22]. The stainless steel needle remained in place for $10 \mathrm{~min}$ after injection to minimize diffusion of the tracer along the needle tract. After completion of injection procedure, the needle was removed, the scalp was sutured, and the rats were replaced in their home cages.

\section{Immunohistochemical tissue processing}

After a survival period of 3-4 days, the animals were deeply anesthetized with sodium pentobarbitone and perfused via the cardiac-vascular system with normal saline followed by fixative consisting of $4 \%$ paraformaldehyde (Merck, Frankfurt, Germany) in $0.1 \mathrm{M}$ phosphate buffer (PB), $\mathrm{pH}$ 7.4. The brains were then rapidly removed and placed in the same fixative at $4{ }^{\circ} \mathrm{C}$ for $3-4$ $\mathrm{h}$ and stored overnight in cold $\left(4{ }^{\circ} \mathrm{C}\right) 0.1 \mathrm{M} \mathrm{PB}$. The brains were then transferred to $30 \%$ sucrose in $0.01 \mathrm{M}$ PB for cryoprotection. Serial saggital brainstem sections (50 $\mu \mathrm{m}$ thick) containing the A7 area and parabrachial nucleus were cut using a frozen sectioning technique. To visualize $\mathrm{BDA}$, free-floating sections were incubated in $A B C$ reagent (Vectastain $A B C$ Peroxidase kit, Vector Labs, Burlingame, CA, USA) overnight at $4{ }^{\circ} \mathrm{C}$. Following rinsing in $0.3 \%$ Triton $\mathrm{X}-100$ in phosphate buffered saline (TPBS) and in phosphate buffer (PB), BDA injection was visualized by the dark Nickel $(\mathrm{Ni})$ - diaminobenzidine (DAB) reaction: $0.05 \%$ DAB (DAB, Sigma, St. Louis, MO, USA) containing $0.01 \% \mathrm{H}_{2} \mathrm{O}_{2}$ and $0.04 \%$ nickel ammonium sulphate in $0.1 \mathrm{M} \mathrm{PB}$ for $10 \mathrm{~min}$. The reaction was terminated by extensive washes in PB. For visualization of noradrenergic neurons in the A7 area, sections were incubated in $2 \%$ bovine serum albumin and $10 \%$ normal goat serum for $1 \mathrm{~h}$ and subsequently in anti-rabbit tyrosine hydroxylase $(\mathrm{TH}$; diluted 1:3000) overnight at $4{ }^{\circ} \mathrm{C}$. The $\mathrm{TH}$ antibody was used here because we found that it stained dendritic structures better than that of the dopamine- $\beta$-hydorxylase antibody (DBH; Chemicon, Temecula, CA, USA) which was used for post hoc immunostaining of neurons after electrophysiology recording (see below). After rinsing with TPBS, the sections were incubated in biotinylated secondary antibody (diluted 1:200) at room temperature for $1 \mathrm{~h}$. After several rinses in TPBS and in PB, immunoreactivity for TH was visualized by the red Nova Red reaction (Vector Labs) for 1-2 min at room temperature. For each brain, all sections were mounted on slides and alternate series were counterstained with cresyl violet to highlight cytoarchitonic divisions; the other sections were dehydrated in ethanol and coverslipped with DPX (Sigma).

\section{Electrophysiology}

Sprague-Dawley rat pups of both sexes, aged 8-10 days, were used. They were anaesthetized with $5 \%$ isoflurane in pure oxygen and decapitated. Their brains rapidly exposed and chilled with ice-cold artificial cerebrospinal fluid 
(ACSF) consisting of (in $\mathrm{mM}$ ): $119 \mathrm{NaCl}, 2.5 \mathrm{KCl}, 1.3$ $\mathrm{MgSO}_{4}, 26.2 \mathrm{NaHCO}_{3}, 1 \mathrm{NaH}_{2} \mathrm{PO}_{4}, 2.5 \mathrm{CaCl}_{2}$, and 11 glucose, oxygenated with $95 \% \mathrm{O}_{2}$ and $5 \% \mathrm{CO}_{2}, \mathrm{pH}$ 7.4. Sagittal brainstem slices $(300 \mu \mathrm{m})$ containing the trigeminal motor nucleus (Mo5) and A7 area were cut using a vibroslicer (D.S.K. Super Microslicer Zero 1, Dosaka EM, Kyoto, Japan) and were kept in an interface-type chamber at room temperature $\left(24-25{ }^{\circ} \mathrm{C}\right)$ for at least $90 \mathrm{~min}$ to allow recovery.

Slices were transferred to an immersion-type recording chamber mounted on an upright microscope (BX51WI, Olympus Optical Co., Ltd., Tokyo, Japan) and were continuously perfused with oxygenated ACSF at $2-3 \mathrm{ml} / \mathrm{min}$. Neurons were viewed using Nomarski optics; those located about $200 \mu \mathrm{m}$ rostral to the anterior border of Mo5 and having a large cell body (diameter about $20-25 \mu \mathrm{m}$ ) were considered to be NAergic A7 neurons [12-14] and were used for recordings (Fig. 3a). The patch pipettes, pulled from borosilicate glass tubing $(1.5 \mathrm{~mm}$ outer diameter, $0.32 \mathrm{~mm}$ wall thickness; Warner Instruments Corp., Hamden, CT, USA), had a resistance of about 3-5 $\mathrm{M} \Omega$ when filled with internal solution consisting of (in $\mathrm{mM}$ ): $131 \mathrm{Cs}-$ gluconate, $20 \mathrm{CsCl}, 10 \mathrm{HEPES}, 2$ EGTA, $8 \mathrm{NaCl}, 2$ ATP, and $0.3 \mathrm{GTP} ; \mathrm{pH}$ adjusted to 7.2 with $\mathrm{CsOH}$. Recordings were made at room temperature $\left(24-25^{\circ} \mathrm{C}\right)$ with a patch amplifier (Multiclamp $700 \mathrm{~A}$; Axon Instruments Inc.; Union City, CA, USA) in voltage-clamp mode. The membrane potential $(\mathrm{Vm})$ was clamped at $-70 \mathrm{mV}$ and a voltage step of $5 \mathrm{mV}$ was applied at $0.1 \mathrm{~Hz}$ throughout the recording to monitor serial resistances and the data were discarded if the values varied by more than $20 \%$ of the original value, which was usually less than $20 \mathrm{M} \Omega$. Signals were low-pass filtered at a corner frequency of $2 \mathrm{kHz}$ and digitized at $10 \mathrm{kHz}$ using a Micro 1401 interface running Signal software (Cambridge Electronic Design, Cambridge, UK) for episode-based capture recording. To elicit synaptic activity, a constant current pulse $(25-500 \mu \mathrm{A} ; 100 \mu \mathrm{s})$ was delivered every $10 \mathrm{~s}$ through a bipolar stainless steel electrode (FHC, Bowdoinham, ME 04008 USA). To isolate excitatory postsynaptic currents (EPSCs) 1 $\mu \mathrm{M}$ strychnine plus $100 \mu \mathrm{M}$ picrotoxin (Ptx) were added to ACSF. All data are presented as the mean \pm standard error of the mean (SEM) and were compared using the paired $t$ test. The criterion for significance was a $p$ value $<0.05$.

In all experiments, $6.7 \mathrm{mM}$ biocytin was routinely included in the internal solution to fill the recorded neurons (Fig. 3a). Neurons were filled by passive diffusion of biocytin from the patch pipette during the recording period, without application of current. After recording, the pipettes were withdrawn and the slices fixed overnight at $4{ }^{\circ} \mathrm{C}$ in $4 \%$ paraformaldehyde (Merck) in $0.1 \mathrm{M}$ phosphate buffer ( $\mathrm{PB}, \mathrm{pH} 7.4)$, then rinsed with $\mathrm{PB}$ several times, and subjected to immunohistochemistry (IHC) procedures without further sectioning. Briefly, the slices were rinsed in phosphate-buffered saline containing $0.03 \%$ Triton X-100 (PBST), then incubated for $1 \mathrm{~h}$ at room temperature in PBST containing $2 \%$ bovine serum albumin and $10 \%$ normal goat serum. The slices were then incubated overnight at $4{ }^{\circ} \mathrm{C}$ in PBST containing a 1/1300 dilution of mouse antibody against rat DBH (Chemicon, Temecula, CA, USA) and a 1/200 dilution of avidin-AMCA (Vector Labs). After PBST rinses, the slices were incubated for $2 \mathrm{~h}$ with tetramethylrhodamine isothiocyanate (TRITC)- conjugated goat anti-mouse IgG antibodies (Jackson, Pennsylvania, USA) diluted $1 / 50$ in PBST, then observed under a fluorescence microscope (Aioplan 2, Zeiss, Oberkochen, Germany) or a confocal microscope (Leica TCS SP5, Hamburger, Germany) for the identification of cell types (Fig. 1c-e).

All chemicals used to prepared ACSF and pipette solution preparation and $\mathrm{Ba}^{2+}$ were from Merck; Ptx, strychnine, and biocytin were from Sigma; and DL2-amino-5-phosphonopentanoic acid (AP5), 6,7-dinitroquinoxaline- 2,3-dione (DNQX), DAMGO and naloxone were from Tocris-Cookson (Bristol, UK).

\section{Results}

\section{BDA Injection sites}

The localization of injection sites was examined and only experiments that had an injection site within the LPB area were accepted for further investigation. An example of an accepted injection site is shown in Fig. 1a, b. An overlay of camera lucida drawings of all sections with BDA deposits (Fig. 1a, b) shows that spreading of the injected BDA was restricted to the LPB area in this case. The injection sites (as indicated by the tissue damaged by the cannula tip) were centered on the central lateral (cl) LPB (Fig. 1c-h), and the injected BDA extended to the lateral crescent (lcr), external medial (em) and external lateral (el) subnuclei, as labeled neurons and neuritis were identified in these areas (Fig. 1e-h). Some fibers in the superior cerebellar peduncle (scp) were also labeled, but labeled neurons in the deep cerebellar nuclei were not observed. Similar injection site locations and trace spreading patterns were observed in the other animal. The injection sites of these two cases were compatible with previous studies $[19,21]$ and the observations described below were based on these two cases.

\section{Anterograde labeled axons in the $\mathbf{A} 7$ cell group}

In sections that comprise BDA injection sites and the trigeminal motor nucleus (Mo5), a cluster of $\mathrm{TH}-$ immunoreactive (TH-ir) neurons located rostral to the 


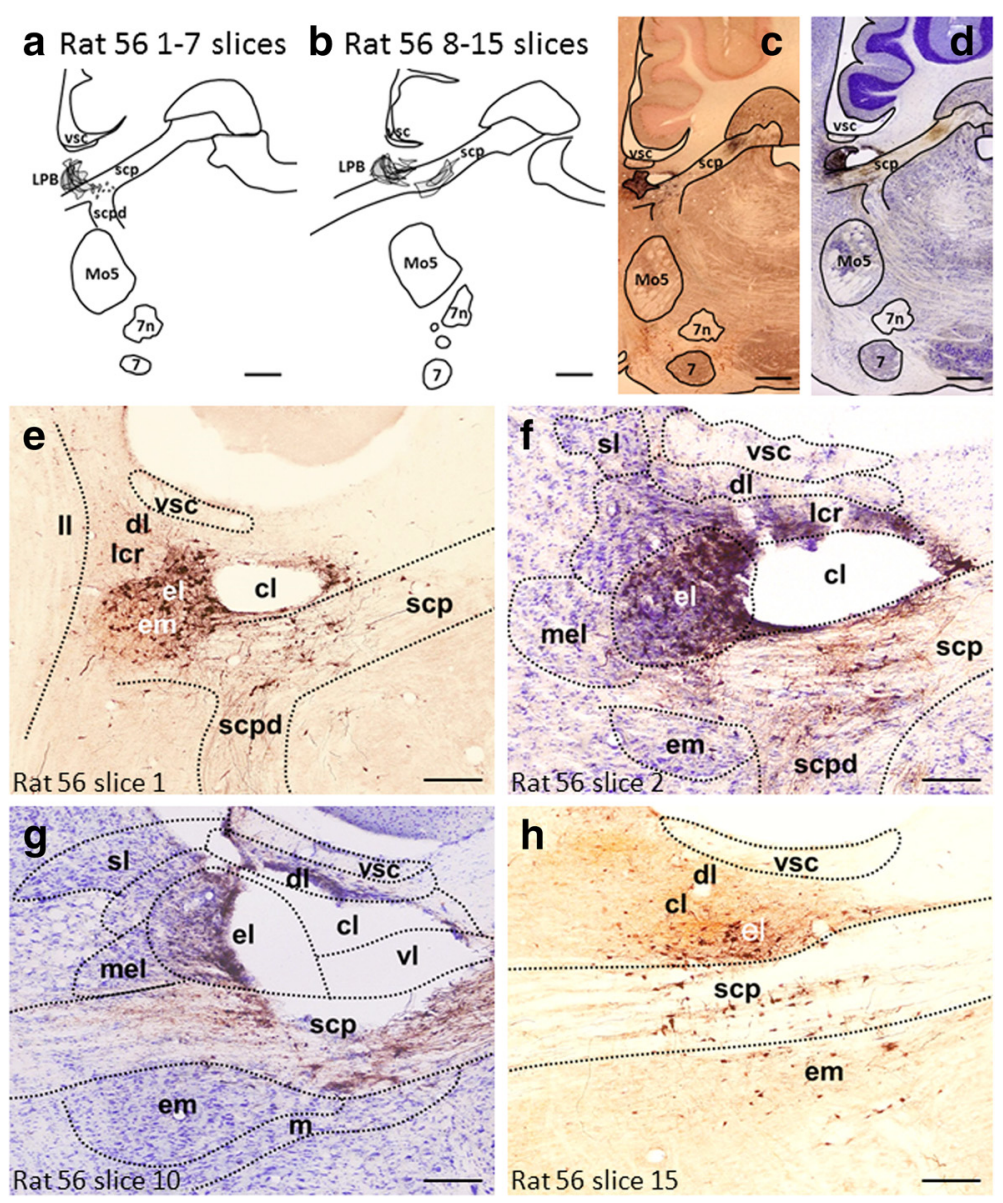

Fig. 1 BDA injection sites. a \& $\mathbf{b}$ Camera lucida drawings from saggital brainstem sections that contain a BDA deposit in the parabrachial nucleus (a \& b). c-g Microphotographs of a BDA injection site are shown in low-power (c: Nova Red; d: Nissl-stained) and magnified views (e \& $\mathbf{h}$ : Nova Red; $\mathbf{f} \& \mathbf{g}$ : Nissl-stained) The nomenclature for the divisions of subdivisions of the parabrachial nucleus was adapted from Sarhan et al. [21]. Note that the injection site is centered in the central lateral (cl) subnuclus of the parabrachial nucleus. $\mathrm{Cl}$, central lateral subnucleus; dl, dorsal lateral subnucleus; el, external lateral subnucleus; em, external medial subnucleus; Icr, lateral crescent subnucleus; II, lateral lemniscus; LPB, lateral parabrachial nucleus; m, medial parabrachial nucleus; mel, external lateral subnucleus of the mesencephalic parabrachial nucleus; Mo5, trigeminal motor nucleus; scp, superior cerebellar peduncle; scpd, superior cerebellar peduncle, descending limb; sl, superior lateral subnucleus of the mesencephalic parabrachial nucleus; vl, ventral lateral subnucleus; vsc, ventral spinocerebellar tract; $7 \mathrm{n}$, facial nerve; 7 , facial nucleus. Scale $\operatorname{bar}=500 \mu \mathrm{m}(\mathbf{a}-\mathbf{d}), 150 \mu \mathrm{m}(\mathbf{e}-\mathbf{h})$

Mo5 could be labeled with an antibody to TH using Nova Red as the chromogen (Fig. 2a). This group of $\mathrm{TH}$-ir neurons has soma of a multipolar shape (Fig. 2b) and is presumed to be NAergic A7 neurons. BDA deposits in the LPB produced antetrograde labeling of axons among cell bodies (Fig. 2a) and dendrites (Fig. 2d, f) of NAergic A7 neurons. These labeled axonal terminals with varicosity-like structures were stained dark blue by nickel intensified DAB histochemical procedures (see Fig. 2e, g). As the TH-ir elements were stained brown-red using Nova Red as the chromogen, they could be easily distinguished from the BDA-labeled axonal terminals. As can be seen (Fig. 2c, e, g), many swollen BDA-labeled varicosities and end-terminations could be clearly identified, some of these being in close proximity to soma (Fig. 2b, c), proximal and distal dendrites of NAergic A7 neurons (Fig. 2d-g).

\section{Characterization of LPB-A7 EPSCs}

To further confirm that the above observed physical contacts between BDA-labeled varicosities and TH-ir soma and dendrites are functional synaptic contacts, we made whole cell recordings from NAergic A7 neurons in rats aged 8-10 days and examined whether extracellular stimulation to the LPB could evoke synaptic currents in NAergic A7 neurons. The electrophysiological criteria for recordings from NAergic A7 neurons have been described previously $[12,13]$. Briefly, we made recordings in current-clamp mode and depolarizing/ hyperpolarizing current pulses were injected to check 

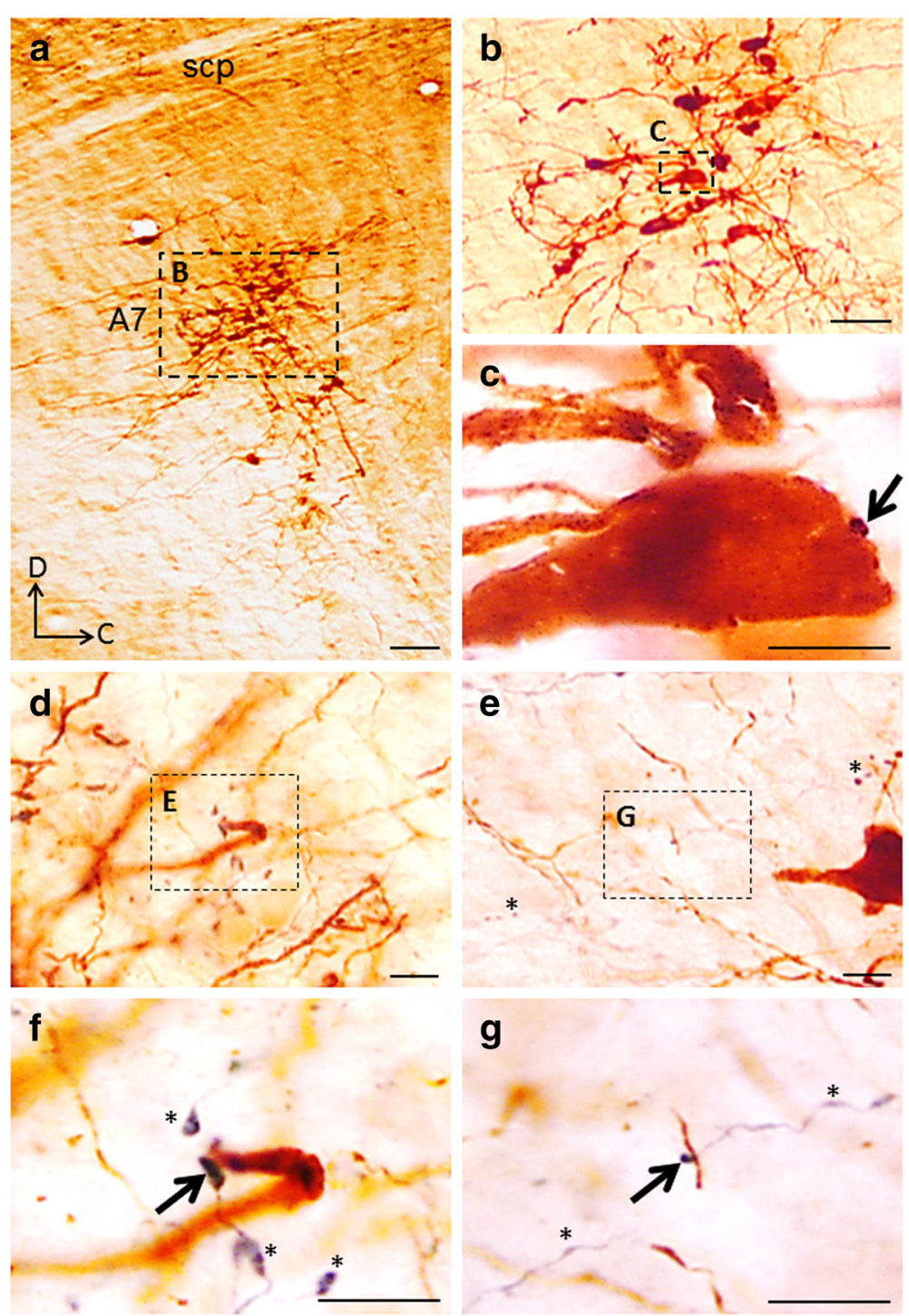

Fig. 2 BAD-labeled axonal terminals in the A7 area. a. Light microscopic photograph of a rat brainstem section showing BDA deposit in the parabrachial nucleus and TH-ir neurons in the A7. Insert (b) shows a photograph of TH-ir neurons in the A7 with high power. Inserts (c), (e) and (g) show high power photographs of axonal terminals in the A7 area as indicated by dotted squares in the (b), (d) and (f), respectively. Note the terminals of BDA-labeled fibers with prominent en passant type varicosities (asterisks) and the contacts of terminals on large soma in (c), and dendrites in (e, g) as indicated by arrows. d, dorsal; scp, superior cerebellar peduncle. Scale bar = $100 \mu \mathrm{m}(\mathbf{a}), 50 \mu \mathrm{m}(\mathbf{b}), 10 \mu \mathrm{m}(\mathbf{c}-\mathbf{g})$

the firing and membrane properties of the recorded neurons. Neurons that displayed neither a voltage sag nor rebound action potentials on injection of a hyperpolarizing current pulse, but displayed a voltage-dependent delay in initiation of the first AP on injection of depolarizing current pulses were used for subsequent voltage-clamp recordings (see [12, 13]). All of the recorded neurons showing above physiological criteria were further confirmed to be DBH-ir by post hoc IHC staining (Fig. 3a).

Under voltage-clamp recording with $\mathrm{Vm}$ clamped at $-70 \mathrm{mV}$ and blockade of GABAergic and glycinergic synaptic transmission by addition of $0.1 \mathrm{mM}$ picrotoxin and $1 \mu \mathrm{M}$ strychnine into the bath medium, extracellular stimulation of the trigeminal motor nucleus (Mo5), superior cerebellar peduncle (scp) and 7th nerve could not evoke detectable synaptic currents in NAergic A7 neurons until the stimulating intensity was increased up to $500 \mu \mathrm{A}$, when inward currents of small amplitude were elicited (Fig. 3b, c). In contrast, large and inward synaptic currents were evoked with a small stimulating intensity (threshold: $30 \mu \mathrm{A}$ ) in an intensity-dependent manner when the stimulating electrode was positioned in the LPB area (Fig. 3b, c). The synaptic currents evoked by LPB stimulation were blocked by bath application of $10 \mu \mathrm{M} C \mathrm{NQX}$, a non-NMDA receptor blocker 

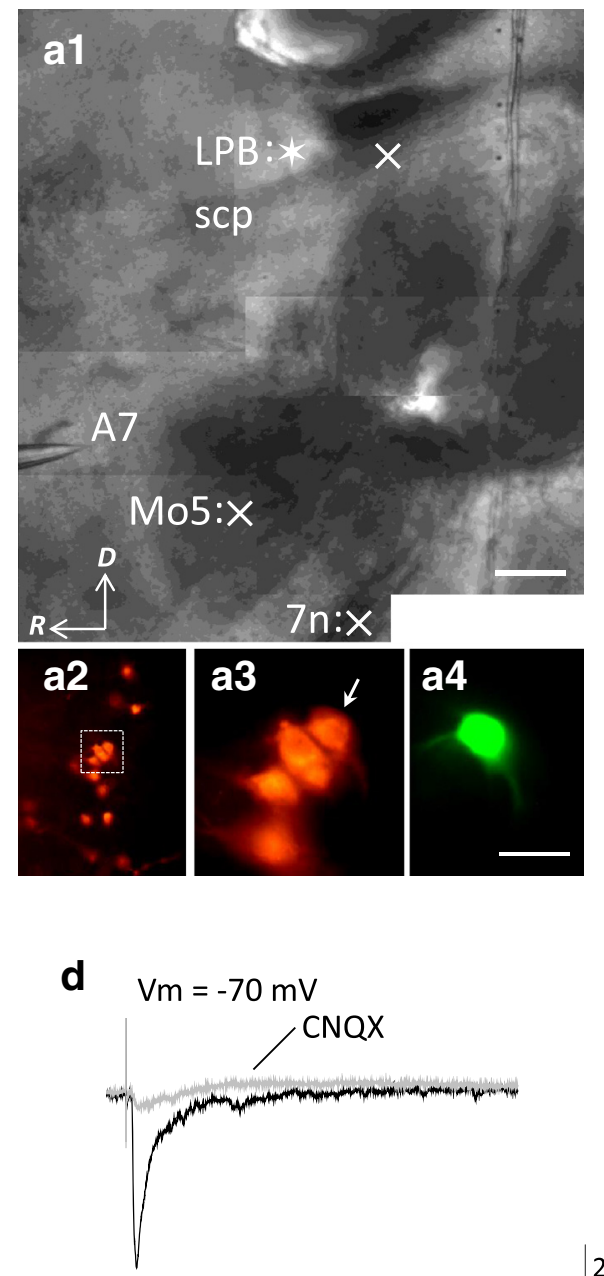

b
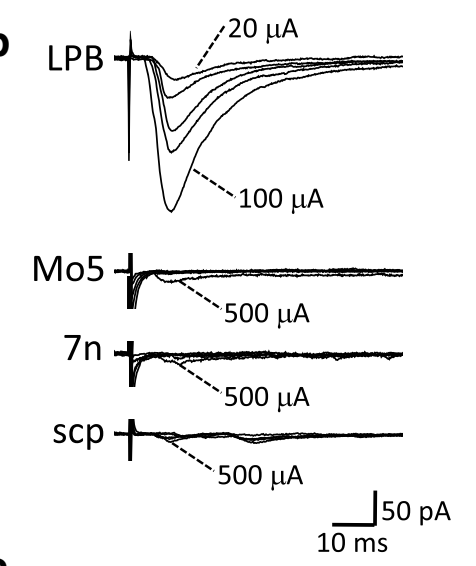

C
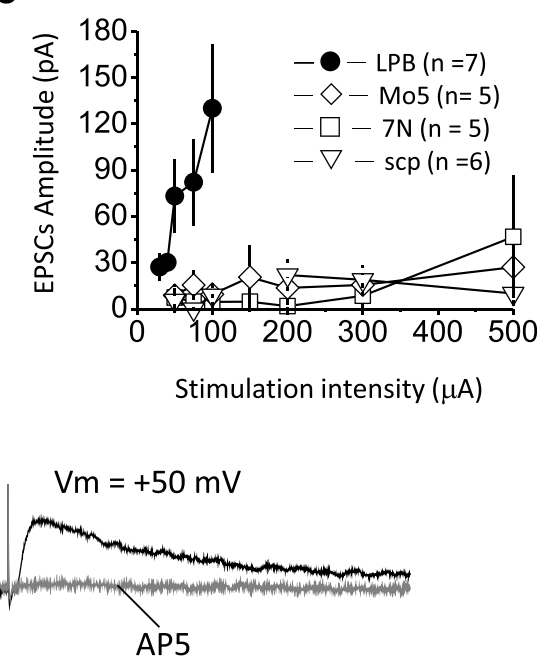

$$
\underset{50 \mathrm{~ms}}{\mathrm{~m}} \mathrm{pA}
$$

Fig. 3 LPB-A7 EPSCs. a. Photographs of a Nomaskic image of a living saggital brainstem slice for electrophysiology recording (A1) and of a fluorescent image of post hoc immunostaining after recording (A2-A3). The asterisk marks the position of extracellular stimulation of the LPB and symbols $\times$ mark the positions of stimulation of the scp: superior cerebellar peduncle $(n=5)$, Mo5: trigeminal motor nucleus (Mo5) and 7n: the 7th (A1). A cluster of TH-ir neurons (A2, A3) was identified in A7: A7 catecholamine cell group (see glass pipette in A1), which was $~ 200$ mm rostral to the anterior border of the Mo5. One of the TH-ir neurons (see arrow in A3) was recorded and filled with biocytin (A4). D: dorsal; R: rostral. b. Representative experiments show recording of EPSCs in NAergic A7 neurons responding to extracellular stimulation of the LPB (upper traces), Mo5 (middle upper traces), 7n (middle lower traces), and scp (bottom traces). c. Summarized results of the experiments shown in $B$. Note that, with a stimulating intensity $<100 \mu \mathrm{A}$, EPSCs were evoked only when the stimulating electrode was placed in the LPB. Parentheses indicate the number of experiments conducted for testing LPB $(n=7)$, Mo5 $(n=5), 7 n(n=50$ and $\operatorname{scp}(n=6)$ stimulation. d. Representative experiments show that LPB-A7 EPSCs were blocked by CNQX $(\mathrm{Vm}=-70 \mathrm{mV})$ and by AP5 $(\mathrm{Vm}=+50 \mathrm{mV})$. Scale bar $=250 \mu \mathrm{m}(\mathrm{A} 1), 30 \mu \mathrm{m}(\mathrm{A} 2-\mathrm{A} 4)$

(Fig. 3d, left traces); clamping Vm at $+50 \mathrm{mV}$ in the subsequent recording revealed an outward current that was blocked by bath application of $50 \mu \mathrm{M}$ AP5, a selective NMDA receptor blocker (Fig. 3d, right traces). These results show that the excitatory postsynaptic currents (EPSCs) evoked by LPB stimulation in NAergic A7 neurons (referred as LPB-A7 EPSCs) were glutamatergic, with glutamate acting at both non-NMDA and NMDA receptors. The latency, $10-90 \%$ rise time, half width of
LPB-A7 EPSCs was $3.3 \pm 2.3 \mathrm{~ms}, 1.7 \pm 0.2 \mathrm{~ms}$ and $8 \pm$ $0.7 \mathrm{~ms}$, respectively.

\section{Presynaptic modulation of $\mu$-receptor on LPB-A7 EPSCs}

Given that LPB neurons have been shown to express large amounts of $\mu$-opioid receptors at their axonal terminals to modulate the release of neurotransmitters $[23,24]$, activation of these presynaptic $\mu$-opioid receptors at terminal from LPB are expected to have a 
presynaptic modulation on the evoked EPSCs if the activity was indeed resulted from LPB stimulation. We therefore examined the effect of $\mu$-opioid receptor activation on the amplitude and paired-pulse ratio (PPR) of LPB-A7 EPSCs. Bath application of 0.5 $\mu$ M DAMGO, a selective $\mu$-opioid receptor agonist, significantly attenuated the amplitude of LPB-A7 EPSCs to $47.5 \pm 12.6 \%$ ( $n=5$ cells, $p<0.01$, paired- $t$ test) of the baseline level (Fig. 4a, b). Subsequent application of $5 \mu \mathrm{M}$ naloxone, a selective $\mu$-receptor blocker, significantly reversed the effect of DAMGO; the EPSC amplitude was reversed to $86.7 \pm 13.1 \%$ of the baseline level (Fig. $4 \mathrm{a}$, b), showing that effect of DAMGO application was due to activation of $\mu$-receptors. In addition to reducing the EPSC amplitude, the effect of DAMGO was associated with an increase in PPR, an indicator of presynaptic effect [25]. The LPB-A7 EPSCs was evoked with a pair of pulses with $50 \mathrm{~ms}$ of inter-pulse interval and the PPR was measured as ratio of amplitude of EPSCs evoked by the second pulse to that by the first pulse. The PPR in control conditions was $1.51 \pm 0.6$ and was increased to $2.99 \pm 1.32$ ( $n=9$ cells, $p<0.01$, paired- $t$ test $)$ upon DAMGO application (Fig. 4c, d), indication a decrease in probability of glutamate release upon $\mu$-receptor activation. These results show that activation of $\mu$ receptors inhibits LPB-A7 synaptic transmission (Fig. 4a, b), which involved a presynaptic modulation (Fig. 4c, d). They further support the argument that the evoked EPSCs are specific to LPB stimulation.

\section{Discussion}

The results of the studies described in this report provide morphological and electrophysiological evidence for the existence of a monosynaptic connection between neurons in the lateral parabrachial nucleus and noradrenergic neurons in the A7 catecholamine cell
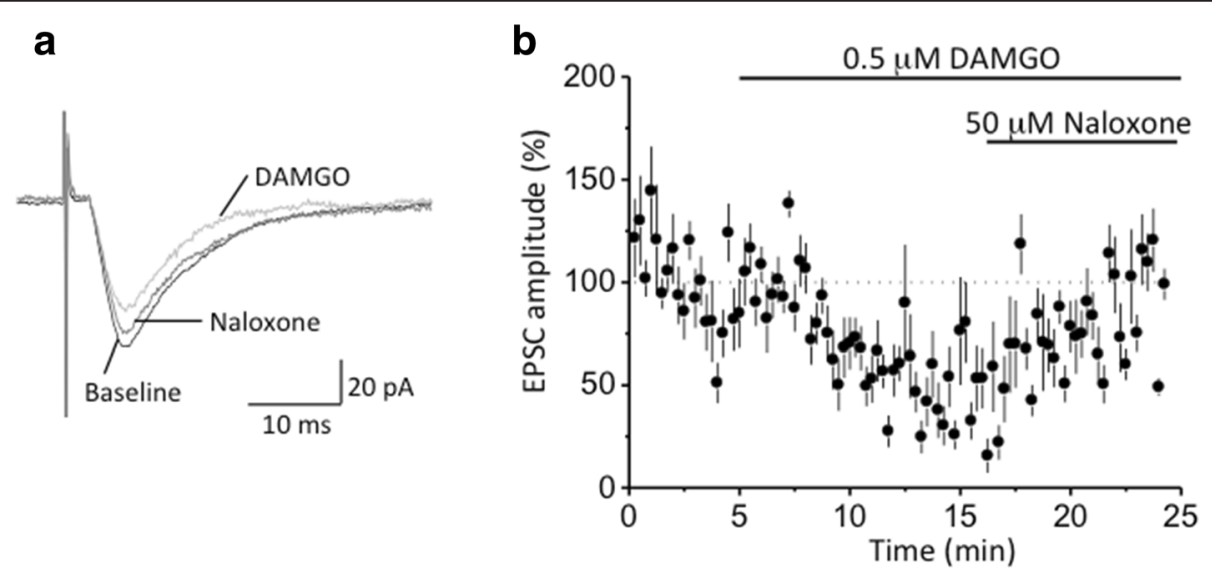

C

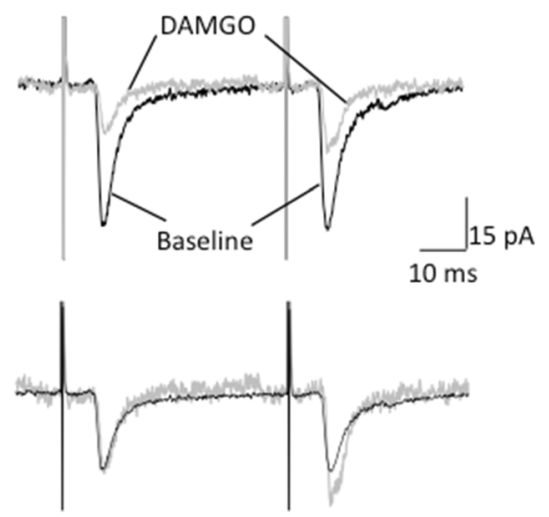

d

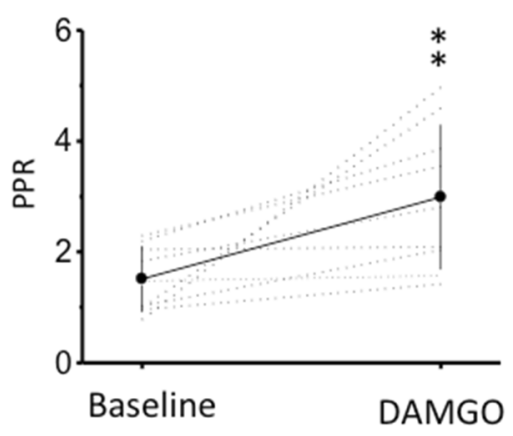

Fig. 4 Presynaptic modulation of LPB-A7 EPSCs by $\mu$-opioid receptors. a. Representative experiment showing that the amplitude of LPB-A7 EPSCS was attenuated by DAMGO, and the effect was reversed by subsequent application of naloxone. $\mathbf{b}$. Summarized results of the experiments shown in $\mathbf{a}$. The amplitude of the EPSCs was normalized to the averaged value of the baseline recording (see dotted line). $\mathbf{c}$. Representative experiment showing the effect of DAMGO on the PPR of LPB-A7 EPSCs. The upper traces are overlays of raw data traces (black: before and gray: after DAMGO application); note that DAMGO attenuated EPSCs as shown above. The lower traces show normalization of the response evoked by the first pulse of the two traces shown above and reveal an increase in the PPR by DAMGO. $\mathbf{d}$. Summarized results of the experiments shown in c. The dotted lines are the results of individual experiments and the symbol line and vertical line are the mean and standard error, respectively. Asterisks mark significance at $p<0.01$ 
group. We have shown that LPB neurons project to the A7 and target on the soma, the proximal and distal dendrites of NAergic neurons. In addition, we have shown that excitatory synaptic responses evoked in the NAergic A7 neurons by extracellular stimulation of the LPB area were mediated by glutamate acting at both non-NMDA and NMDA receptors. The release of glutamate from the axonal terminal of the LPB neurons is modulated by $\mu$-opioid receptors.

Our anatomical experiments of light microscopic observations show physical contacts of BDA-labeled varicosities on $\mathrm{TH}$-ir elements, including soma and dendritic aborizations in the A7 and suggest the existence of direct synaptic connections between LPB neurons and NAergic A7 neurons. The reliability of such anatomical data depends critically on obtaining a precise $\mathrm{BDA}$ injection site in the LPB. Indeed, our conclusions were drawn from observations made from two animals in which the location of the tip of the injection cannula and deposits of the injected BDA were in the LPB area, though some contamination occurred in the adjacent white-matter structure, the superior cerebellar peduncle (scp; see Fig. 1). This observation of BDA-labeled fibers in the scp raises the possibility that some BDA-labeled buttons in A7 might come from cerebellar deep nuclei but not from the LPB. However, no previous study has ever demonstrated neuronal connection between the cerebellar deep nuclei and the A7 cell group and our electrophysiological results also disfavor this possibility (see below). In addition to those on $\mathrm{TH}$-ir elements, there were also $\mathrm{TH}$-ir varicosities (see asterisks in Fig. 2e)-g) making physical contacts with non-TH-ir postsynaptic elements. In addition to NAergic neurons, there are interneurons scattering over the A7 area and making synaptic contacts with NAergic neurons [12]. It has been suggested that some of these interneurons are GABAergic, and afferents inputs from the periaqueductal gray area (PGA) to the A7 area could indirectly regulate NAergic neurons through regulating of these GABAergic interneurons [26-30]. Accordingly, BDA-labeled varicosities without contacts with $\mathrm{TH}$-ir postsynaptic elements may contact GABAergic interneurons in A7 and afferents from the LPB might also indirectly regulate NAergic A7 neurons through GABAergic interneurons.

Extracellular stimulation of the LPB area evoked EPSCs in NAergic A7 neurons with the intensity threshold being about $30 \mu \mathrm{A}$. In our data pool, EPSCs of large amplitude could be readily induced with a stimulating intensity lower than $100 \mu \mathrm{A}$; in contrast, within the same range of stimulating intensity (30-100 $\mu \mathrm{A}$; see Fig. $3 \mathrm{c}$ ), no detectable EPSCs could be induced when the stimulating electrode was positioned in the scp, Mo5 or 7th nerve. Since the distance of these structures to NAergic A7 neurons are similar to that of LPB to NAergic A7 neurons, especially scp is in vicinity of the LPB, these observations show that the spread of current delivered from the stimulating electrode with an intensity of $<100 \mu \mathrm{A}$ was limited to the local area, so that LPB neurons were activated only when the stimulating electrode was positioned in the LPB but not in its vicinity. These observations also show no functional connection between cerebellar efferent and NAergic A7 neurons, therefore confirming our anatomical observations that DAB-labeled buttons found in A7 were indeed from the LPB but not scp. The inhibition of LPB-A7 EPSCs by DAMGO, a selective $\mu$-opioid receptor, further supports the argument that EPSCs evoked by LPB stimulation were caused by glutamate release from terminals of LPB neurons, because LPB neurons have been shown to express $\mu$-opioid receptors $[23,24]$. Moreover, the effect of DAMGO on EPSC amplitude was associated with an increase in PPR, showing that $\mu$-opioid receptors are located at the axonal terminal of LPB neurons and regulate glutamate release. The EPSCs evoked by local LPB stimulation were blocked by application of non-NMDA and NMDA receptor blockers; these results echo previous studies arguing that the majority of parabrachial neurons are glutamatergic [31, 32]. The features of short delaylatency, rapid and smooth rise phase, of PLB-A7 EPSCs suggest that PLB-A7 EPSCs are monosynaptic. In brain slice preparation at room temperature, the range of latency between electrical stimulation and onset of synaptic responses varies approximately from 2 to $4 \mathrm{~ms}$ among different synapses; for example, the latency of monosynaptic unitary EPSCs is described as $4 \mathrm{~ms}$ for mossy fibers on CA3 pyramidal neurons in hippocampus [33] and $3 \mathrm{~ms}$ for local inputs on stellate neurons in visual cortex [34]. Since our recording conditions are similar to these studies, namely using extracellular stimulation and making recording at room temperature, the latency of $3.3 \mathrm{~ms}$ for PLB-A7 EPSCs suggest that the transmission is monosynaptic.

\section{Conclusions}

The above electrophysiological features together with anatomical data all support the argument that LPB input makes direct synaptic contacts with NAergic A7 neurons. Regarding to the physiological role, however, whether this connection is involved in regulation of nociception requires further evidences. This is because LPB receives not only ascending projections from nociceptive neurons in dorsal horn but also from the medial portion of the nucleus of the solitary tract, which conveys signals from many visceral receptors, such as baroreceptors and cardiopulmonary receptors, and from gustatory receptors [35-38]. Due to the limitation of 
experimental material (brain slice) used in this study, we were unable to specify whether the stimulated LPB neurons in brain slice or the BDA labeled LPB neurons in the tracing experiments were nociceptive. Nevertheless, as LPB is the major central target for the ascending nociceptive signal from the dorsal spinal cord [39-41] and NAergic A7 neurons forms a part of descending analgesia system by projecting their axonal terminals to the dorsal spinal cord, our results do suggest the possibility that noxious stimuli could excite NAergic A7 neurons, one of the component of descending NAergic system, through with the LPB-NAergic A7 connection; namely, LPB-NAergic A7 connection could function as a negative feedback control loop for pain regulation. Our results also show that LPB-NAergic synaptic transmission is regulated by presynaptic $\mu$-opioid receptors. As neurons in the A7 catecholamine cell group (both NAergic and interneurons) receive enkephalin and other endogenous opioids projection from the rostroventromedial medulla and PGA [26, 27, 29], the efficiency and operation of LPB-NAergic A7 synapses, a possible feedback control loop for pain regulation, could be modulated by the other components of descending analgesic systems, such PAG and RVM.

\section{Abbreviations}

cl: Central lateral subnucleus; dl: Dorsal lateral subnucleus; el: External lateral subnucleus; em: External medial subnucleus; Icr: Lateral crescent subnucleus; II: Lateral lemniscus; LPB: Lateral parabrachial nucleus; m: Medial parabrachia nucleus; mel: External lateral subnucleus of the mesencephalic parabrachial nucleus; Mo5: Trigeminal motor nucleus; scp: Superior cerebellar peduncle; scpd: Superior cerebellar peduncle, descending limb; sl: Superior lateral subnucleus of the mesencephalic parabrachial nucleus; vl: Ventral lateral subnucleus; vsc: Ventral spinocerebellar tract; 7n: Facial nerve; 7: Facial nucleus.

\section{Competing interests}

The authors declare that they have no competing interests.

\section{Authors' contributions}

CY Liu and ML Lee collected and analyzed morphological and physiological data, respectively. HW Yang formed the conception and design of the experiments and wrote the manuscript along with MY Min, CS Yang and CM Chen. All authors read and approved the final manuscript.

\section{Acknowledgements}

This work was supported by grants from the National Science Council, Taiwan, to HWY (NSC 100-2320-B-040-010-MY3).

\section{Author details \\ ${ }^{1}$ Department of Life Sciences, and Agricultural Biotechnology Center, National Chung Hsing University, Taichung, Taiwan. ²Department of Nursing, Jen-Teh Junior College of Medicine, Nursing and Management, Miaoli, Taiwan. ${ }^{3}$ Department of Biomedical Sciences, Chung Shan Medical University, 110, Chien-Kuo N. Rd, Sec. 1, Taichung 402, Taiwan. ${ }^{4}$ Department of Medical Research, Chung Shan Medical University, Taichung, Taiwan. ${ }^{5}$ Department of Nursing, Hungkuang University, Taichung, Taiwan. ${ }^{6}$ Rong Hsing Research Center for Translational Medicine, and the iEGG Center, National Chung Hsing University, Taichung, Taiwan. ${ }^{7}$ Department of Life Sciences, College of Life Science, National Taiwan University, Taipei, Taiwan.}

Received: 14 April 2015 Accepted: 20 August 2015

Published online: 18 September 2015

\section{References}

1. Pertovaara A. Noradrenergic pain modulation. Prog Neurobiol. 2006;80:53-83.

2. North RA, Yoshimura M. The actions of noradrenaline on neurones of the rat substantia gelatinosa in vitro. J Physiol. 1984;349:43-55.

3. Danzebrink RM, Gebhart GF. Antinociceptive effects of intrathecal adrenoceptor agonists in a rat model of visceral nociception. J Pharmacol Exp Ther. 1990;253:698-705

4. Carlsson A, Falck B, Fuxe K, Hillarp NA. Cellular localization of monoamines in the spinal cord. Acta Physiol Scand. 1964;60:112-9.

5. Clark FM, Proudfit HK. The projection of noradrenergic neurons in A7 catecholamine cell group to the spinal cord in rat demonstrated by antrograde tracing combined immunohistochemistry. Brain Res. 1991;547:279-88

6. Kwiat GC, Basbaum Al. The origin of brainstem noradrenergic and serotonergic projections to the spinal cord dorsal horn in the rat. Somatosens Mot Res. 1992:9:157-73.

7. Proudfit HK, Clark FM. The projection of locus coeruleus neurons to the spinal cord. Prog Brain Res. 1991;88:123-41

8. Westlund KN, Coulter JD. Descending projections of the locus coeruleus and subcoeruleus/medial parabrachial nuclei in monkey: axonal transport studies and dopamine-b-hydroxylase immunocytochemistry. Brain Res Rev. 1980;2:235-64.

9. Holden JE, Schwartz EJ, Proudfit HK. Microinjection of morphine in the A7 catecholamine cell group produces opposing effects on nociception that are mediated by alpha1- and alpha2- adrenoceptors. Neuroscience. 1999;83:979-90.

10. Yeomans DC, Clark FM, Paice JA, Proudfit PH. Antinociception induced by electrical stimulation of spinally projecting noradrenergic neurons in the A7 catecholamine cell group of the rat. Pain. 1992;48:449-61.

11. Dennis SG, Melzack R, Gutman S, Boucher F. Pain modulationby adrenergic agents and morphine as measured by three pain tests. Life Sci. 1980:26:1247-59.

12. Min MY, Wu YW, Shih PY, Lu HW, Lin CC, Wu Y, et al. Physiological and morphological properties of, and effect of substance $P$ on, neurons in the A7 catecholamine cell group in rats. Neuroscience. 2008;153:1020-33.

13. Min MY, Wu YW, Shih PY, Lu HW, Wu Y, Hsu CL, et al. Roles of A-type potassium currents in tuning spike frequency and integrating synaptic transmission in noradrenergic neurons of the A7 catecholamine cell group in rats. Neuroscience. 2010;168:633-45.

14. Min MY, Shih PY, Wu YW, Lu HW, Lee ML, Yang HW. Neurokinin 1 receptor activates transient receptor potential-like currents in noradrenergic A7 neurons in rats. Mol Cell Neurosci. 2008:42:56-65.

15. Wu YW, Wang HW, Lin CC, Lu HW, Cheng SJ, Yang HW, et al. GABA receptor-mediated tonic inhibition of noradrenergic $A 7$ neurons in the cat. J Neurophysiol. 2011;105:2715-28.

16. Sugiyama D, Hur S, Pickering AE, Kase D, Kim S, Kawamata M, et al. Furue, In vivo patch-clamp recording from locus coeruleus neurons in the rat brainstem. J Physiol. 2012;590:2225-31.

17. Takagi H, Shiomi H, Kuraishi Y, Fukui K, Ueda H. Pain and the bulbospinal noradrenergic system: pain-induced increase in normetanephrine content in the spinal cord and its modification by morphine. Eur J Pharmacol. 1979:54:99-107.

18. Tyce GM, Yaksh TL. Monoamine release from cat spinal cord by somatic stimuli: an intrinsic modulatory system. J Physiol. 1981;314:513-29.

19. Bernard JF, Alden M, Besson JM. The organization of the efferent projections from the pontine parabrachial area to the amygdaloid complex: a Phaseolus vulgaris leucoagglutionin (PHA-L) study in the rat. J Comp Neurol. 1993:329:201-29.

20. Gauriau C, Bernard JF. Pain pathways and parabrachial circuits in the rat. Exp Physiol. 2002;87:251-58.

21. Sarhan M, Freund-Mercier MJ, Veinante P. Branching patters of parabrachial neurons projecting to the central extended amgydala: single axonal reconstructions. J Comp Neurol. 2005;491:418-42.

22. Paxinos $G$, Watson C. The Rat brain in stereotaxic coordinates, forth ed. San Diego: Academic; 1998.

23. Ding YQ, Kaneko T, Nomura S, Mizuno N. Immunohistochemical localization of mu-opioid receptors in the central nervous system of the rat. J Comp Neurol. 1996:367:375-402.

24. Mansour A, Fox CA, Burke S, Akil H, Watson SJ. Immunohistochemical localization of the cloned mu opioid receptor in the rat CNS. J Chem Neuroanat. 1995:8:283-305. 
25. Zucker RS, Regehr WG. Short-term synaptic plasticity. Annu Re Physiol. 2002;64:355-405.

26. Bajic D, Proudfit HK. Projections of neurons in the periaqueductal gray to pontine and medullary catecholamine cell groups involved in the modulation of nociception. J Comp Neurol. 1999;405:359-79.

27. Bajic D, Van Bockstaele EJ, Proudfit HK. Ultrastructural analysis of ventrolateral periaqueductal gray projections to the A7 catecholamine cell group. Neuroscience. 2001;104:181-97.

28. Holden JE, Pizzi JA. The challenge of chronic pain. Adv Drug Deliv Rev 2003:55:935-48.

29. Holden JE, Proudfit HK. Enkephalin neurons that project to the A7 catecholamine cell group are located in nuclei that modulate nociception: ventromedial medulla. Neuroscience. 1998;83:929-47.

30. Nuseir K, Proudfit HK. Bidirectional modulation of nociception by GABA neurons in the dorsolateral pontine tegmentum that tonically inhibit spinally projecting noradrenergic A7 neurons. Neuroscience. 2000;96:773-83.

31. Guthmann A, Fritschy JM, Ottersen OP, Torp R, Herbert H. GABA, GABA transporters, GABA(A) receptor subunits, and GAD mRNAs in the rat parabrachial and Kölliker-Fuse nuclei. J Comp Neurol. 1998;400:229-43.

32. Yokota S, Oka T, Tsumori T, Nakamura S, Yasui Y. Glutamatergic neurons in the Kolliker-Fuse nucleus project to the rostral ventral respiratory group and phrenic nucleus: a combined retrograde tracing and in situ hybridization study in the rat. Neurosci Res. 2007:59:341-46.

33. Jonas P, Major G, Sakmann B. Quantal component of unitary EPSCs at the mossy fibre synapses on CA3 pyramidal cells of rat hippocampus. J Physiol. 1993;472:615-63.

34. Stern P, Edwards FA, Sakmann B. Fast and slow components of unitary EPSCS on stellate cells elicited by focal stimulation in slices of rat visual cortex. J Physiol. 1992:449:247-78.

35. Chamberlin NL, Saper CB. Topographic organization of respiratory responses to glutamate microstimulation of the parabrachial nucleus in the rat. J Neurosci. 1994;14:6500-10.

36. Felder RB, Mifflin SW. Modulation of carotid sinus afferent input to nucleus tractus solitarius by parabrachial nucleus stimulation. Circulation Rese. 1988;63:35-49.

37. Jia $H G$, Rao ZR, Shi JW. An indirect projection from the nucleus of the solitary tract to the central nucleus of the amygdala via the parabrachial nucleus in the rat. A light and electron microscopic study. Brain Res. 1994;663:181-90

38. Takayama K, Miura M. Respiratory responses to microinjection of excitatory amino acid agonist in ventrolateral regions of the lateral parabrachial nucleus in the cat. Brain Res. 1993;604:217-23.

39. Craig AD. Distribution of brainstem projections from spinal lamina I neurons in the cat and the monkey. J Comp Neurol. 1995;361:225-48.

40. Hylden JL, Anton F, Nahin RL. Spinal lamina I projection neurons in the rat: collateral innervation of parabrachial area and thalamus. Neuroscience. 1989;28:27-37.

41. Klop EM, Mouton LJ, Hulsebosch R, Boers J, Holstege G. In cat four times as many lamina I neurons project to the parabrachial nuclei and twice as many to the periaqueductal gray as to the thalamus. Neuroscience. 2005;134:189-97.

\section{Submit your next manuscript to BioMed Central and take full advantage of:}

- Convenient online submission

- Thorough peer review

- No space constraints or color figure charges

- Immediate publication on acceptance

- Inclusion in PubMed, CAS, Scopus and Google Scholar

- Research which is freely available for redistribution 